\title{
CONSTRAINED PV PENETRATION LEVEL IN LV DISTRIBUTION NETWORKS BASED ON THE VOLTAGE OPERATIONAL MARGIN
}

\author{
Carlos GONZALEZ \\ Esat/Electa, KU Leuven - Belgium \\ Pieter VINGERHOETS \\ Esat/Electa, KU Leuven - Belgium \\ pieter.vingerhoets@esat.kuleuven.be
}

Esat/Electa, KU Leuven - Belgium
.be sam.weckx@esat.kuleuven.be

\author{
Tom DE RYBEL \\ Esat/Electa, KU Leuven - Belgium \\ tom.derybel@esat.kuleuven.be
}

\author{
Nick EFKARPIDIS \\ Esat/Electa, KU Leuven - Belgium \\ nikolaos.efkarpidis@esat.kuleuven.be \\ Johan DRIESEN \\ Esat/Electa, KU Leuven - Belgium \\ johan.driesen@esat.kuleuven.be
}

\begin{abstract}
In this paper, an approach to the critical photovoltaic penetration level is presented, based on the capacity of a DSO to regulate the voltage in a MV/LV substation by means of Off-Load Tap Changers. The performance of offload tap changers to cope with an increasing amount of $P V$ is investigated, by introducing the concept of the Voltage Operational Margin: the range of voltage where the tap can be set in order to avoid under-voltage or over-voltage.
\end{abstract}

\section{INTRODUCTION}

The historic function of the conventional, radial distribution networks was viewed primarily as the unidirectional electricity transportation from centralized generating units downward to the end users at lower voltages via the distribution feeders. In case of long feeders or resistive feeders, it was likely to incur under-voltage at the feeder end. One solution was to equip the distribution transformer with a tap changer, in order to adjust the ratio between the primary and the secondary voltages of the transformer, manually and off-load. By setting the tap in an upper position, the last houses of the feeder did not see such a low voltage.

Photovoltaic generation units have been largely introduced in the LV distribution network and are expected to increase in the future. This can compromise the voltage regulation in the distribution transformer, which may fulfil the power quality criteria, defined in the EN-50160 standard [1]:

Table 1:Power quality parameter compliance

\begin{tabular}{|l|c|c|r|}
\hline Parameter & min & max & Compliance \\
\hline Voltage magnitude & $0.9 \mathrm{Un}$ & $1.1 \mathrm{Un}$ & $95 \%$ week \\
\cline { 2 - 4 } & $0.85 \mathrm{Un}$ & $1.1 \mathrm{Un}$ & $100 \%$ week \\
\hline
\end{tabular}

(Un: nominal voltage)

As it is operated nowadays the LV distribution network, the increasing penetration level may increase the chances of incurring over-voltage [2] and the rise of the load demand may increase the chances of having under-voltage. Furthermore, these voltage fluctuations need to be compensated. Any voltage regulation technique may fulfil the voltage criteria shown in Table 1, required to comply the power quality constrains.

Some techniques to keep this situation under control are
Power Curtailment, Demand Side Management (DSM) [3], the use of Automatic Voltage Regulators (AVR), active control of On-Load Tap Changers (OLTC) [4], storage systems and some other techniques. At present, most of these techniques are under research and are investigated as experimental cases. In LV distribution networks, the active control of On-Load Tap Changers seems to be the most feasible solution, since it does not imply the active participation of the customers and it can be easily integrated in a so-called "Active SubStation".

The actual MV/LV transformers may be equipped with tap changers in order to control the voltage along the grid. By adjusting the tap changers, the voltage fluctuations and the voltage magnitude can be kept between limits. In consequence, the tap changers must be set above a minimum voltage $\left(\mathrm{V}_{\text {min,tap }}\right)$, in order to avoid under-voltage anywhere in the grid, and also below a maximum operating voltage $\left(\mathrm{V}_{\max , \text { tap }}\right)$, to prevent over-voltage.

Typically, at no-load conditions, DSOs use to set the tap changers slightly above the nominal voltage Un, typ. $230 \mathrm{~V}$, so when the transformer is loaded, the voltage drop across it can be compensated. However, this voltage drop across the transformer varies depending on the total transformer load, so the load current fluctuations may lead to voltage fluctuations along the day.

\section{VOLTAGE OPERATIONAL MARGIN}

Let us define the Voltage Operational Margin (VOM) as the voltage range within which the tap changer can be kept without incurring neither over-voltage nor under-voltage all over the grid, in any node of the network:

$$
\mathrm{VOM}=\mathrm{V}_{\text {max,tap }}-\mathrm{V}_{\text {min,tap }}
$$

In previous work, the effects of an increasing amount of PV on different power quality indicators were investigated [5]. It was shown that long, ramified cable sections and highly populated feeders (around 60 houses), classified as semiurban, were prone to show voltage problems. Also the city feeder, with an average population (around 40 customers) with a long cable section at the beginning (more than $300 \mathrm{~m}$ ) to cover the last houses of a long street, was also prone to show voltage problems.

In [5] other feeder types like rural and urban ones were also studied. Rural feeders in Flanders are short feeders and not very populated, whereas urban feeders are medium length 
ones with average population. The effect of the PV location was also studied, considering PV locations such as at the beginning of the feeder, at the end or randomly along the feeder.

For every specific condition there exists a different VOM. Within this section, the $\operatorname{VOM}\left(\mathrm{V}_{\max , \text { tap }} ; \mathrm{V}_{\text {min,tap }}\right)$ is dependent on the load and the PV penetration level and its location. This defines some curves, found by running load flows.

Figure 1 shows the curves of $\mathrm{V}_{\text {max,tap }}(245 \mathrm{~V})$ and $\mathrm{V}_{\text {min,tap }}$ $(220 \mathrm{~V})$ when more and more PV units are connected starting at the end of the feeder and towards the head of the feeder. Also the VOM is plotted (in grey) for a specific penetration level of $6 \mathrm{PV}$ units. It can be observed that as far as the number of PV units increases, the margin to operate the tap changer is reducing more and more: the operation becomes more complex. In practical terms, the VOM range means that for these specific grid conditions, we can set passively the tap changer in any position between $\mathrm{V}_{\min \tan }$ and $\mathrm{V}_{\text {max tan }}$ and we will not incur voltage problems.

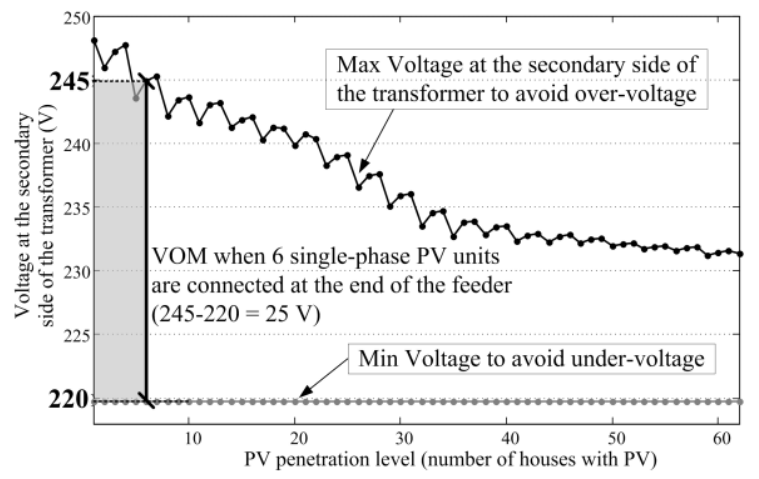

Figure 1: VOM in the semi-urban feeder, PV at the end

Also in Figure 1 can be seen (1) the most unbalanced phase in the grid, since in that phase the effect of the PV units is stronger and (2) adding more and more PV does not affect substantially the minimum voltage $\left(\mathrm{V}_{\text {min,tap }}\right)$ : the minimum voltage depends mainly on how loaded is the feeder.

Connecting more PV units or in a different PV location mean changing the grid conditions, and that is why the VOM range also changes. In Figure 2 and Figure 3, the effect of the PV location in the $\mathrm{V}_{\text {min,tap }}$ and $\mathrm{V}_{\text {max,tap }}$ of two feeders can be seen.

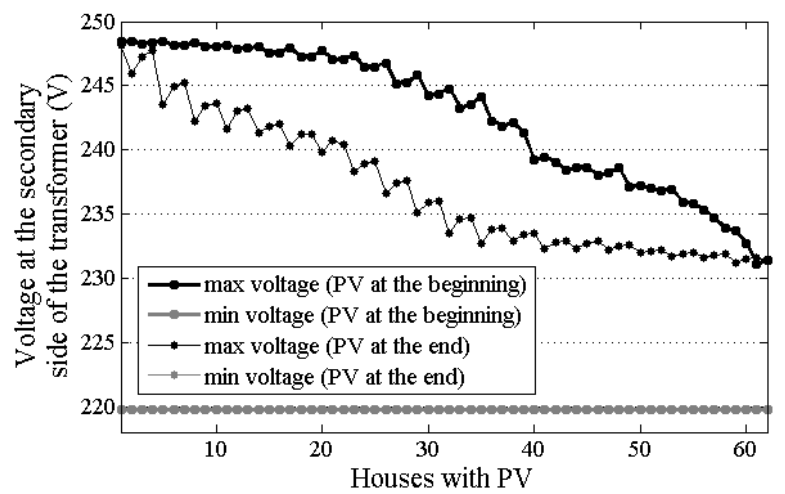

Figure 2: Semi-urban maximum and minimum voltages

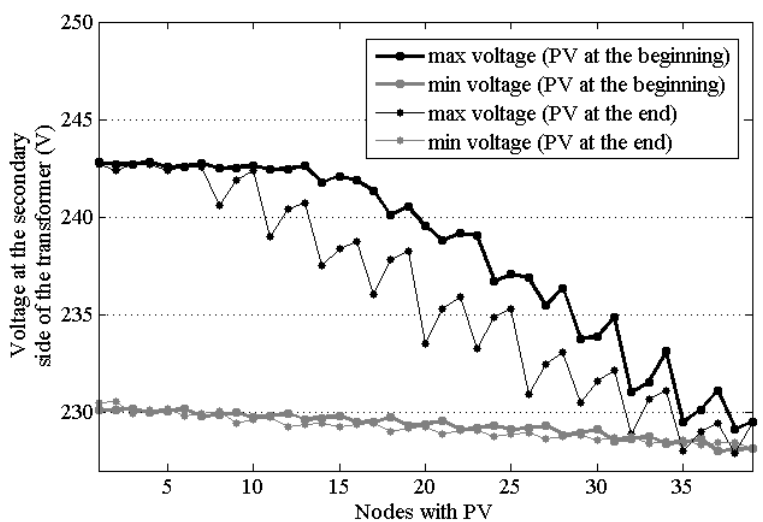

Figure 3: City maximum and minimum voltages

It can be deducted that a large VOM of 10-20V implies an easier voltage control, whereas a small VOM of few Volts may constrain the voltage operation. This last case can be seen in Figure 3 at high PV penetration levels, where the maximum voltage goes below the minimum voltage. This is a critical penetration level: the operation with passive means is no longer feasible, thus claiming for voltage control strategies and/or other active counter-measures.

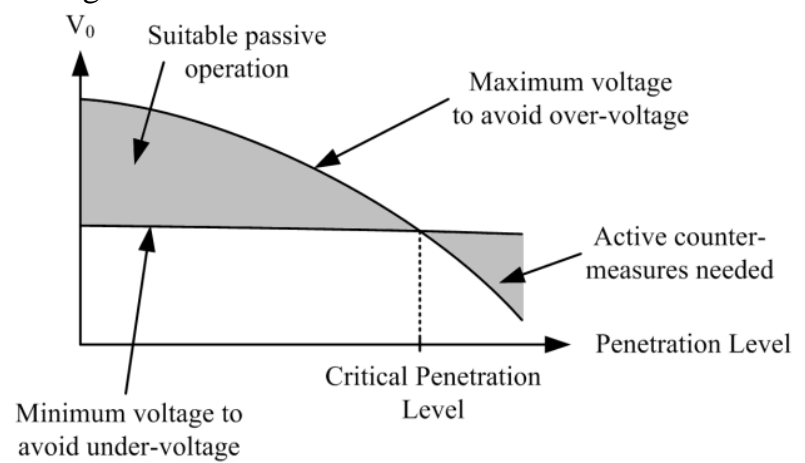

Figure 4: Interpretation of the VOM

When more load is connected to the grid, this case may lead to lower voltages all over the grid. Thus, the tap changer cannot be set so low as before, meaning that $\mathrm{V}_{\text {min,tap }}$ rises. The maximum voltage is basically not affected by a load increase, however, a critical load level can also be seen in Figure 4.

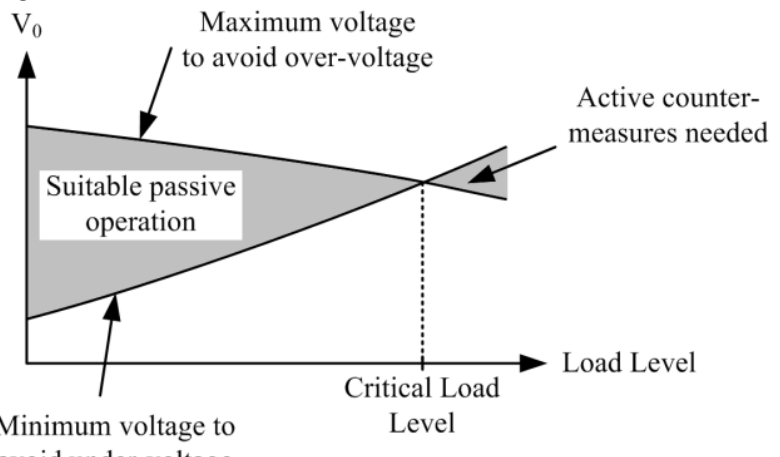
avoid under-voltage

Figure 5: VOM when the load increases, without PV 
For load increases (like electric car penetration) beyond the critical load level point, active measures for load control such as Demand Side Management and coordinated charge of the electric vehicle are required.

The combined two phenomena (load increase and rising PV penetration level) may result in an earlier critical point. The first phenomenon leads to a decreasing $\mathrm{V}_{\text {max,tap }}$, and the second one, to a rising $\mathrm{V}_{\min , \text { tap. }}$. For instance, a combination of an uncoordinated charge of the electrical vehicle and a not regulated PV connection may be a feasible scenario where this would happen. The result is that active countermeasures are required faster than expected.

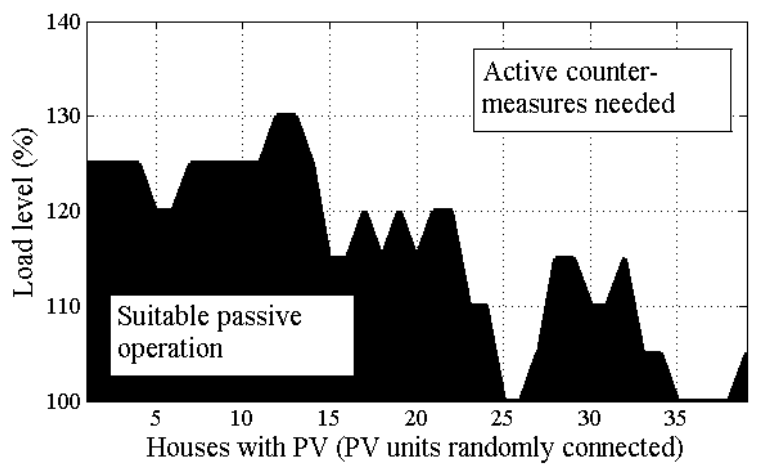

Figure 6: City feeder active/passive operation boundary

\section{MV/LV SUBSTATIONS WITH MORE FEEDERS}

MV/LV substations include normally several feeders, about 5 to 7 feeders in low-populated areas, even 10-13 in highly populated areas. The voltage regulation in the substations is unique for all the feeders together, which implies that the tap setting must satisfy the voltage constraints of all the feeders at the same time. Furthermore, each feeder may have different PV penetration levels, located randomly.

Let's consider a MV/LV substation with 4 feeders (1 city feeder, 1 urban, 1 semi-urban and 1 rural), and each feeder with a different number of PVs, connected randomly. Table 2 shows the PV level for each feeder and the VOM for each feeder calculated individually. If the tap changer is set above $240 \mathrm{~V}$, the semi-urban feeder may have over-voltage somewhere in that feeder. If the tap is below $229 \mathrm{~V}$, the city feeder may have under-voltage in one or more nodes. These become critical feeders.

Table 2: VOM for each feeder and the whole substation

\begin{tabular}{|l|r|l|l|r|}
\hline Feeder & PVs & Vmax & Vmin & VOM \\
\hline City & 12 & $242,4 \mathrm{~V}$ & $\mathbf{2 2 9 , 8 V}$ & $12,6 \mathrm{~V}$ \\
\hline Urban & 24 & $246,4 \mathrm{~V}$ & $211,5 \mathrm{~V}$ & $34,9 \mathrm{~V}$ \\
\hline Semi-urban & 20 & $\mathbf{2 3 9 , 4 V}$ & $219,7 \mathrm{~V}$ & $19,7 \mathrm{~V}$ \\
\hline Rural & 14 & $245,3 \mathrm{~V}$ & $217,6 \mathrm{~V}$ & $27,7 \mathrm{~V}$ \\
\hline Substation & $\mathbf{( 7 0 )}$ & $\mathbf{2 3 9 , 4 V}$ & $\mathbf{2 2 9 , 8 V}$ & $\mathbf{9 , 6 V}$ \\
\hline
\end{tabular}

The result is a substation with a Voltage Margin constrained by the lowest maximum voltage and the highest minimum voltage of all the feeders. In figure 6 it is plotted the VOM curves for each feeder, and the constrained VOM for the whole substation (the grey area).

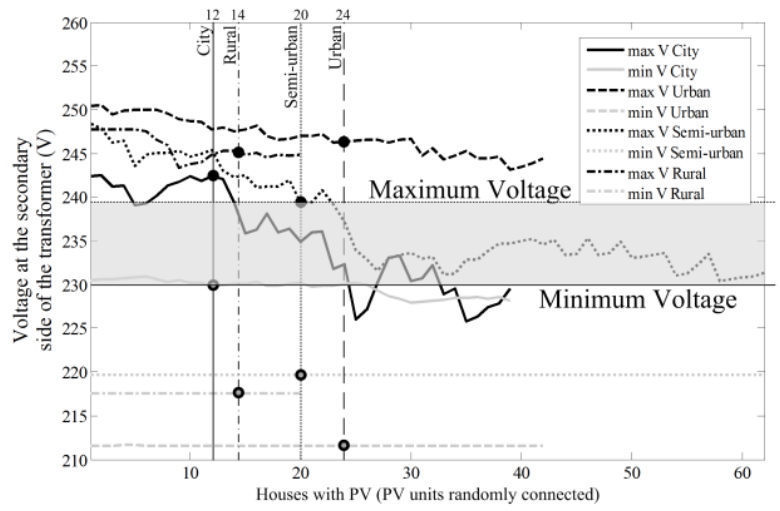

Figure 7: VOM of a substation of 4 feeders

It can be seen that the larger the PV penetration level in the semi-urban or city feeders, the smaller the margin. It has to be considered, as well, that voltage fluctuations in the MV grid can lead to a voltage variations in the LV busbar about $5 \mathrm{~V}$ during the day. Moreover, in simulation, it has been found that variations on how the loads are connected in the grid can lead to a voltage variation of $3-4 \mathrm{~V}$ at most. If these two effects are taken into account, the voltage margin would be strongly reduced.

\section{REVERSE CURRENT INJECTION}

The reverse current flow (to the MV grid) happens when the total amount of generation is larger than the total amount of load in the grid (plus the losses). The effect of the reverse current is the opposite of the load current: a voltage rise. This effect should be taken into account in the voltage regulation in the MV side of the transformer.

Consider a distribution transformer Dy $10 / 0,4 \mathrm{kV}$. The Voltage Margin obtained in the LV side can be translated into a Voltage Margin referred to the MV level, by using the voltage transformation rate: $\mathrm{r}=\mathrm{U}_{\mathrm{n} 1 \text {, line }} / \mathrm{U}_{\mathrm{n} 2 \text {, phase }}=43,48$. For the previous example, the Voltage Margin referred to the LV side was $\left(229,8 \mathrm{~V}_{\text {phase }} ; 239,4 \mathrm{~V}_{\text {phase }}\right)$, whereas the margin referred to the MV side is $\left(9,991 \mathrm{kV}_{\text {line }} ; 10,409 \mathrm{kV}_{\text {line }}\right)$.

Given the MV nominal voltage (10 kV in this case), if the current direction is towards the load (forward), the voltage drop across the transformer impedance leads to a voltage in the LV side slightly below the nominal (for instance 228 $\mathrm{V}_{\text {phase}}$ ), whereas for the same MV nominal voltage, if the current is reverse, it leads to a higher LV voltage (for instance $232 \mathrm{~V}_{\text {phase }}$ ).

The voltage drop across the transformer, $\Delta \mathrm{V}_{\text {trafo }}$, is given by the expression: $\Delta \mathrm{V}_{\text {trafo }}= \pm \mathrm{Z}_{\text {trafo }} * \mathrm{I}_{\text {trafo }}$, where $\mathrm{Z}_{\text {trafo }}$ is the transformer impedance and $\mathrm{I}_{\text {trafo }}$ is the total current flowing 
through the transformer and the sign \pm refers to the direction of the current: forward (+) or reverse (-). Thus, the relation between the voltage drop and the current is linear through the transformer impedance.

The regulation in some countries limits or does not allow the reverse current that can flow through the transformer back to the MV grid. If the reverse current is not limited, it can reach the nominal value. This case may only happen under massive generation. However, the fact that the voltage drop can be of both signs, positive and negative, implies that the passive voltage regulation must work in both scenarios at the same time, adding another restriction in the Voltage Margin. This situation is plotted in Figure 8.

Figure 8 should be read from left to right. It can be seen: (1) the VOM referred to the LV side of the transformer is in the left side of the picture, (2) the VOM is referred to the MV level thanks to the voltage transformation rate $r$, (3) the transformer voltage drop depends on the transformer current $\mathrm{I}_{\text {trafo }}$ (horizontal axis), and plotted for forward and reverse current direction, for the maximum and the minimum tap changer voltages. The shadowed region highlights the suitable passive operation, where the forward and reverse currents can be regulated in a passive way.

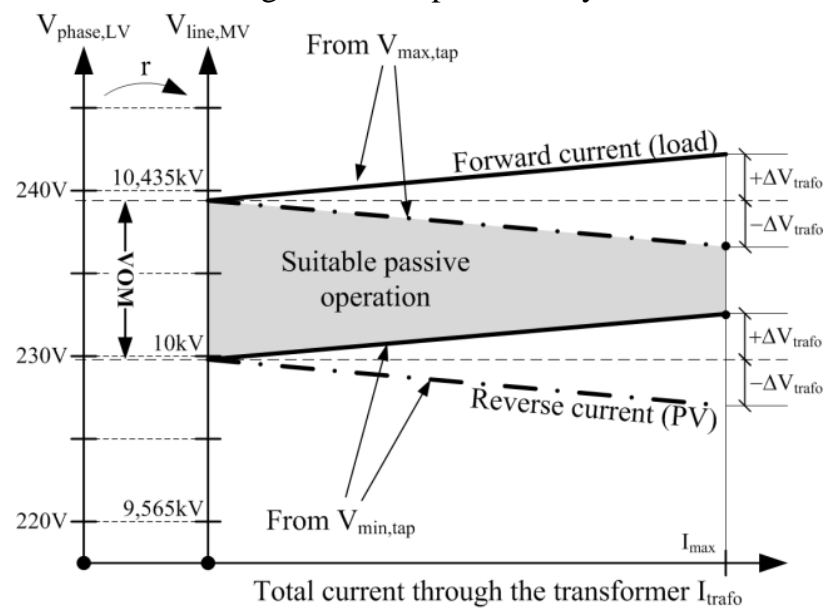

Figure 8: VOM in the MV side depending on the transformer current, (reverse current not limited)

To better understand the figure, let's consider that we set the tap changer in the MV side (if it is the tap type) at 10 $\mathrm{kV}$. Let's also consider the case of maximum current through the transformer $I_{\max }$ (extreme value in the horizontal axis). Looking at the VOM when the reverse current is maximum, this tap position is suitable because it fits between the maximum and the minimum voltages (parallel dotted-segment lines). However, looking at the forward current VOM, this tap position falls below the margin, which may imply problems of under-voltage in the LV network in those moments of maximum forward current.

\section{CONCLUSIONS}

In this paper it has been presented a methodology to estimate the critical PV penetration level and the critical load level beyond which active measures in the LV distribution grid may be required. It is done by analysing the margin left to the DSOs to operate the tap changers (VOM), to avoid over-voltage or under-voltage problems all over the supplied grid with an unspecified time scope. A centralized voltage control (for instance a tap changer in the MV/LV substation) requires to take into account all the feeders together.

Concerning the PV influence, if it is connected at the beginning of the feeder, for low/medium PV penetration levels, the VOM is larger than if the PVs are connected at the end of the feeder. That means deferring the need of active voltage control measures in the LV grid (the passive operation). In the contrary, the combination of factors, (1) the load increase, (2) more DG, (3) MV grid voltage fluctuations and (4) the reverse power flow may reduce drastically the operational margin, thus accelerating the need of active measures in the distribution grid.

\section{AKNOWLEDGEMENT}

The work is supported by the Flemish Government through the LINEAR project (IWT/090800) organized by the Institute for Science and Technology (IWT). S. Weckx has a PhD fellowship of the Research Foundation - Flanders VITO (FWO - VITO).

\section{REFERENCES}

[1] Standard NEN-EN 50160, 2010, Voltage characteristics of electricity supplied by public electricity networks.

[2] T. Vu Van, A. Woyte, F. Harris, L. De Gheselle, G. Palmers, J. Neyens, F. Truyens, B. Bletterie, H. Brunner, K. De Brabandere, J. Reekers, M. Sporleder, B. Blazic, I. Papic, R. Engelen, J. Alenus, 2009, "The Meta PV Project: Photovoltaics for Active Distribution Systems", $24^{\text {th }}$ European Photovoltaic Solar Energy Conference, EU-PVSEC.

[3] D. S. Kirschen, 2003, "Demand-Side View of Electricity Market", IEEE Transactions On Power Systems, vol. 18, No. 2, 2003.

[4] C. Oates, A. Barlow, V. Levi, 2007, "Tap Changer For Distributed Power", 19th International Conference on Electricity Distribution, CIRED 2007.

[5] C. Gonzalez, J. Geuns, S. Weckx, T. Wijnhoven, P. Vingerhoets, T. De Rybel, J. Driesen, 2012, "LV Distribution Network Feeders in Belgium and Power Quality Issues due to Increasing PV Penetration Levels", 3rd IEEE PES Innovative Smart Grid Technologies Europe Conference, IEEE PES-ISGT 2012. 